\title{
Complete Subvarieties of Moduli Spaces and the Prym Map
}

\author{
Carel Faber and Gerard van der Geer
}

\section{Introduction}

In this paper we present a formula for the number of isomorphism classes of $p$-rank zero étale double covers of genus 2 curves over an algebraically closed field of characteristic $p>2$. The formula is a byproduct of our search for complete subvarieties of moduli spaces of curves. Many moduli spaces are not complete because the objects that they parametrize can degenerate. Examples are the moduli spaces of polarized abelian varieties and the moduli spaces of curves. For a given quasi-projective variety knowledge of the maximum dimension of a complete subvariety gives information on its geometry and cohomology and it is a kind of measure how far the variety in question is from being affine. Moreover, in the case of a moduli space it tells us when complete families must degenerate.

For the moduli space $\mathcal{M}_{g} \otimes k$ of curves of genus $g \geq 2$ over a field $k$ one has the upper bound $g-2$ for the dimension of a complete subvariety due to Diaz in characteristic 0 and Looijenga in general, but it is not known how good this bound is. For the moduli space $\mathcal{A}_{g} \otimes k$ of principally polarized abelian varieties of dimension $g$ over $k$ one has an upper bound for the dimension of a complete subvariety $(\operatorname{dim} \leq g(g-1) / 2)$ and it is known that this bound is sharp if the characteristic of $k$ is positive. Recently, Keel and Sadun $[\mathrm{KS}$, Cor.1.2] proved that in char. 0 the bound is not sharp for $g \geq 3$. In characteristic $p>0$ the bound is attained by the complete subvariety of abelian varieties of $p$-rank 0 . This suggests to look at the moduli space of curves in positive characteristic and to impose conditions on the $p$-rank of their Jacobians in order to find complete subvarieties. In characteristic $p>2$ we get a subvariety of $\mathcal{M}_{g} \otimes k$ by considering curves $C$ of genus $g$ together with an étale double cover $C^{\prime}$ whose $p$-rank is 0 ; its expected dimension is $g-2$. Unfortunately, this doesn't yield a complete subvariety in general, because there are components that are not complete. We hope that for almost all primes $p$ there are complete components and then these are necessarily of dimension $g-2$ and show that Diaz's bound is sharp. This necessitates a careful analysis of the components which we haven't carried out yet. We did the analysis for genus 2, where we have a formula for the number of pairs $\left(C^{\prime}, C\right)$ for which the $p$-rank of $C^{\prime}$ is zero. The number is zero for $p=3$, but positive for $p \geq 5$. One of our main tools is the use of tautological classes.

The contents of this paper are as follows. We prove that in characteristic $p>0$ the locus of stable curves of $p$-rank $\leq f$ is pure of codimension $g-f$ in $\overline{\mathcal{M}}_{g} \otimes k$. Then we consider the Prym map and analyze it using tautological classes. We study the locus of 
curves with an étale double cover of $p$-rank 0 in some detail. In particular, in genus 2 we obtain a formula for the number of such curves. We end with several examples illustrating our formula.

We shall use the following notation throughout this paper. For nonnegative integers $g$ and $n$ such that $2 g-2+n>0$, we denote by $\mathcal{M}_{g, n}$ the moduli space of $n$-pointed curves of genus $g$, by $\overline{\mathcal{M}}_{g, n}$ the Deligne-Mumford compactification of $\mathcal{M}_{g, n}$, and by $\widetilde{\mathcal{M}}_{g, n}$ the moduli space of stable $n$-pointed curves of genus $g$ whose dual graph is a tree; often these curves are referred to as curves of compact type. All these moduli spaces are smooth algebraic stacks defined over $\mathbb{Z}$. We recall that on an $n$-pointed curve the $n$ points are distinct, ordered, nonsingular points. We can view $\widetilde{\mathcal{M}}_{g, n}$ as an open substack of $\overline{\mathcal{M}}_{g, n}$ and $\mathcal{M}_{g, n}$ as one of $\widetilde{\mathcal{M}}_{g, n}$. When $n=0$ (thus $g \geq 2$ ), we write $\mathcal{M}_{g}, \overline{\mathcal{M}}_{g}$, and $\widetilde{\mathcal{M}}_{g}$. We have

$\overline{\mathcal{M}}_{g}=\mathcal{M}_{g} \cup \cup_{i=0}^{[g / 2]} \Delta_{i}$, where $\Delta_{0}$ denotes the divisor of irreducible nodal curves and their degenerations and $\Delta_{i}$ for $i>0$ denotes the divisor of reducible curves with components of genus $i$ and $g-i$, and their degenerations. Moreover, we have $\overline{\mathcal{M}}_{g}-\widetilde{\mathcal{M}}_{g}=\Delta_{0}$. By $\mathcal{A}_{g}$ we denote the moduli space of principally polarized abelian varieties of dimension $g$ and by $\mathcal{A}_{g}^{*}$ the Satake compactification constructed by Faltings.

In the following we shall work over an algebraically closed field $k$ and we shall often write $\mathcal{M}_{g}\left(\overline{\mathcal{M}}_{g}, \widetilde{\mathcal{M}}_{g}\right.$, etc. $)$ instead of $\mathcal{M}_{g} \otimes k\left(\overline{\mathcal{M}}_{g} \otimes k, \widetilde{\mathcal{M}}_{g} \otimes k\right.$, etc. $)$. Since the stacks $\overline{\mathcal{M}}_{g, n}$ and $\mathcal{A}_{g}$ are smooth, the codimension of an intersection is at most the sum of the codimensions. We use this fact without comment in $\S 2$ and $\S 4$.

If $k$ is of characteristic $\neq 2$ then we denote by $R \mathcal{M}_{g}$ (resp. $R \widetilde{\mathcal{M}}_{g}$ ) the moduli space of pairs $\left(C^{\prime}, C\right)$ of connected étale double covers $C^{\prime} \rightarrow C$ of a genus $g$ curve $C$, with $C$ nonsingular (resp. of compact type). The curves $C^{\prime}$ have genus $2 g-1$. Such covers correspond exactly to points of order 2 in the Jacobian of $C$.

\section{$\S 1$. Complete subvarieties and tautological classes}

It is a well-known result of Diaz [D1, Thm.4] that a complete subvariety of the moduli space $\mathcal{M}_{g} \otimes k$ of curves of genus $g \geq 2$ has dimension $\leq g-2$ (the extension to positive characteristic is due to Looijenga [L, p.412]). But for $g \geq 4$ it is not known whether this bound is sharp. A similar question occurs for the moduli space $\mathcal{A}_{g} \otimes k$ of principally polarized abelian varieties of dimension $g$. It is known by [G, Cor.2.7] that a complete subvariety of $\mathcal{A}_{g} \otimes k$ has codimension at least $g$ (we recall the proof below). However, here one knows that in characteristic $p>0$ the moduli space $\mathcal{A}_{g} \otimes \mathbb{F}_{p}$ contains a complete codimension $g$ subvariety, namely the locus of abelian varieties of $p$-rank zero, cf. [Ko, Thm.7]. As to characteristic 0, Keel and Sadun $\left[\mathrm{KS}\right.$, Cor.1.2] recently showed that $\mathcal{A}_{g} \otimes \mathbb{C}$ does not contain a codimension $g$ complete subvariety for $g \geq 3$, as had been conjectured by Oort [O3, 2.3G]. Thus the maximum dimension of a complete subvariety of $\mathcal{A}_{g} \otimes k$ depends on the characteristic of $k$ ! (It is not known what the maximum dimension of a complete subvariety of $\mathcal{A}_{g} \otimes \mathbb{C}$ is for $g>3$.)

Recall the definition of the tautological ring associated to $\mathcal{A}_{g}$. The moduli space $\mathcal{A}_{g}$ carries a tautological bundle, the Hodge bundle $\mathbb{E}$. It is defined by specifying for every principally polarized abelian scheme $X$ over $S$ with zero-section $s$ a rank $g$ vector bundle on $S: s^{*} \Omega_{X / S}^{1}$. The Chern classes $\lambda_{i}=c_{i}(\mathbb{E})$ of this bundle are called tautological classes and 
they generate a $\mathbb{Q}$-subalgebra $T^{*}\left(\mathcal{A}_{g}\right)$ of the Chow ring $A^{*}\left(\mathcal{A}_{g}\right) \otimes \mathbb{Q}$, called the tautological subring.

It is shown in $\left[\mathrm{G}\right.$, Thm.2.1] that the relations $\operatorname{ch}_{2 k}(\mathbb{E})=0$ hold $\forall k \geq 1$ in $T^{*}\left(\mathcal{A}_{g}\right)$, hence $T^{*}\left(\mathcal{A}_{g}\right)$ is a quotient of $T_{g}$, the cohomology ring of the compact dual of the Siegel upper half space:

$$
T_{g}=\mathbb{Q}\left[\lambda_{1}, \ldots, \lambda_{g}\right] /\left(\operatorname{ch}_{2}(\mathbb{E}), \ldots, \operatorname{ch}_{2 g}(\mathbb{E})\right) .
$$

Note that the vanishing of the first $g$ even $\mathrm{ch}_{2 k}$ implies the vanishing of all even $\mathrm{ch}_{2 k}$ (and in fact also the vanishing of the odd $\mathrm{ch}_{2 k+1}$ for all $k \geq g$ ).

Hence $T_{g}$ is a complete intersection ring with socle in degree $\left(\begin{array}{c}g+1 \\ 2\end{array}\right)$. In [G, Prop.2.2] it is proved that $\lambda_{g}=0$ in $T^{*}\left(\mathcal{A}_{g}\right)$, so $T^{*}\left(\mathcal{A}_{g}\right)$ is a quotient of $T_{g-1}$. It then follows that there are no further relations: combine the fact that the $p$-rank zero locus $V_{0}$ in $\mathcal{A}_{g} \otimes \overline{\mathbb{F}}_{p}$ is a complete subvariety of pure codimension $g$ (dimension $\left(\begin{array}{l}g \\ 2\end{array}\right)$ ) with the ampleness of $\lambda_{1}$ on $\mathcal{A}_{g} \otimes \overline{\mathbb{F}}_{p}$ due to Moret-Bailly [M-B, p.181] (so $\lambda_{1}^{\left(\begin{array}{l}g \\ 2\end{array}\right)} \neq 0$ in every characteristic, and a generator of the socle of $T_{g-1}$ doesn't vanish on $\mathcal{A}_{g}$ ). To summarize the above:

$$
T^{*}\left(\mathcal{A}_{g}\right) \cong T_{g-1}
$$

This implies that $\lambda_{1}^{\left(\begin{array}{c}g \\ 2\end{array}\right)+1}=0$ and hence that a complete subvariety of $\mathcal{A}_{g}$ has codimension at least $g$.

For a more complete discussion see [FL, §11] and [GO, §3], but note that Thm. 3.4 in $[\mathrm{GO}]$ is at present only a conjecture, unfortunately.

\section{$\S 2$. Subvarieties of the moduli space of curves defined by the $p$-rank}

We are interested in the loci of curves whose $p$-rank is 0 . For a smooth curve $C$ of genus $g$ over an algebraically closed field $k$ of characteristic $p>0$ we define the $p$-rank $f$ of $C$ as the $p$-rank of its Jacobian $J$ : if $J[p]$ denotes the kernel of multiplication by $p$ on $J$ then

$$
\# J[p](k)=p^{f} .
$$

We have $0 \leq f \leq g$. Alternative definitions of this $p$-rank are: the semisimple rank of the $\sigma$-linear operator $F$ induced by the relative Frobenius on $H^{1}\left(J, O_{J}\right)$ (or on $H^{1}\left(C, O_{C}\right)$ ); or dually, the semisimple rank of the $\sigma^{-1}$-linear Cartier operator $V$ on $H^{0}\left(J, \Omega_{J}^{1}\right.$ ) (or on $\left.H^{0}\left(C, \Omega_{C}^{1}\right)\right)$.

To extend this definition to stable curves we consider a family $C \rightarrow B$ of stable curves of genus $g$ over an irreducible base scheme $B$. We let $\omega_{C / B}$ be the relative dualizing sheaf and write $\mathbb{E}=\pi_{*}\left(\omega_{C / B}\right)$ for the Hodge bundle, a locally free sheaf of rank $g$. On it we have the relative Cartier operator $V: \mathbb{E} \rightarrow \mathbb{E}$. For a point $b \in B$ the $p$-rank of the fibre $C_{b}$ is defined as the semisimple rank of $V$ on the fibre $\mathbb{E}_{b}$. Equivalently, we can look at the action of the relative Frobenius on $R^{1} \pi_{*} O_{C}$. A result of Grothendieck (see [Ka, Th.2.3.1]) says that the $p$-rank is lower semi-continuous on $B$.

The following lemma is a variant of a lemma of Oort, see [O1, 1.6]. The proof is similar to that of Oort and is omitted. 
(2.1) Lemma. Let $B$ be an irreducible scheme over $k$ and $C \rightarrow B$ a stable curve over $B$. Let $f$ be the $p$-rank of the generic fibre $C_{\eta}$. Let $W \subset B$ denote the closed set over which the p-rank of the fibre is at most $f-1$. Then either $W$ is empty or $W$ is pure of codimension 1 in $B$.

(2.2) Definition. We define the locus $V_{f}\left(\overline{\mathcal{M}}_{g}\right)$ as the locus of curves with $p$-rank $\leq f$ in $\overline{\mathcal{M}}_{g}$; similarly, we define $V_{f}\left(R \widetilde{\mathcal{M}}_{g}\right)$ as the locus of pairs $\left(C^{\prime}, C\right)$ for which the $p$-rank of $C^{\prime}$ is $\leq f$. We denote by $R V_{0}\left(\widetilde{\mathcal{M}}_{g}\right)$ the locus of pairs $\left(C^{\prime}, C\right)$ for which the $p$-rank of $C$ is 0 . Similarly, we denote by $V_{f}\left(\mathcal{A}_{g}\right)$ the locus of principally polarized abelian varieties of dimension $g$ with $p$-rank $\leq f$.

We have the following result.

(2.3) Theorem. The locus $V_{f}\left(\overline{\mathcal{M}}_{g}\right)$ is pure of codimension $g-f$ in $\overline{\mathcal{M}}_{g}$.

Proof. We apply Lemma 2.1 just as in [Ko, p.164]: Fix $r<g$. Let $C_{r}$ be any component of $V_{r}\left(\overline{\mathcal{M}}_{g}\right)$. Let $C_{r+1}$ be a component of $V_{r+1}\left(\overline{\mathcal{M}}_{g}\right)$ containing $C_{r}$. Note that a priori it is possible that $C_{r}=C_{r+1}$. We obtain a sequence $C_{r} \subseteq C_{r+1} \subseteq \ldots \subseteq C_{g}=\overline{\mathcal{M}}_{g}$. The lemma tells us: for $r^{\prime}=r, r+1, \ldots, g-1$, if $C_{r^{\prime}} \neq C_{r^{\prime}+1}$, then $C_{r^{\prime}}$ has codimension one in $C_{r^{\prime}+1}$. We conclude: the codimension of $C_{r}$ is at most $g-r$.

Now let $r=0$. As is well-known, $C_{0}$ is a complete subvariety of $\widetilde{\mathcal{M}}_{g}$ (the $p$-rank of a generalized abelian variety is at least its torus rank). On the other hand, we have Diaz's upper bound $2 g-3$ for the dimension of such a complete subvariety; see Lemma 2.4 below. It follows that $C_{0}$ has codimension $g$ : the case $f=0$ of the theorem.

If every $C_{r}$ would contain a $C_{0}$, we would now be done. Since we don't know how to establish this, we proceed differently. We prove that every $C_{r}$ has codimension $g-r$ in $\overline{\mathcal{M}}_{g}$ by induction on $r$. The case $r=0$ has been established, so assume $r>0$. Since $\operatorname{codim}\left(C_{r}\right) \leq g-r \leq g-1$, the component $C_{r}$ cannot be complete in $\widetilde{\mathcal{M}}_{g}$, hence intersects $\Delta_{0}$. The intersection consists of points corresponding to curves of genus $g-1$ and $p$ rank $\leq r-1$ with two points identified. The dimension of the intersection is at most $2(g-1)-3+(r-1)+2=2 g-4+r$ : this is obvious for $g=2$ and follows for $g \geq 3$ by induction. Hence the dimension of $C_{r}$ is $2 g-3+r$.

Remark. It is not clear whether $V_{f}\left(\mathcal{A}_{g}\right)$ intersects the Torelli image of $\widetilde{\mathcal{M}}_{g}$ transversally. Also, is for example $V_{0}\left(\overline{\mathcal{M}}_{g}\right)$ irreducible for $g \geq 4$ ?

The theorem we just established is the analogue for the moduli space of curves of a result of Koblitz [Ko, Thm. 7] for the moduli of principally polarized abelian varieties and of the main result of Norman and Oort [NO, Thm.4.1] for abelian varieties with arbitrary polarization.

The following result was already observed by Diaz [D2, p.80].

(2.4) Lemma. The dimension of a complete subvariety of $\widetilde{\mathcal{M}}_{g, n}$ is at most $2 g-3+n$.

Proof. This is trivial for $g \leq 1$. For $g \geq 2$, it suffices to prove this for $n=0$. By the DiazLooijenga bound $g-2$ for the dimension of a complete subvariety of $\mathcal{M}_{g}$, we may assume that the complete subvariety $Y$ meets some $\Delta_{i}$, with $0<i \leq g / 2$. Then by induction $\operatorname{dim} Y \leq 1+(2 i-2)+(2(g-i)-2)=2 g-3$. 
(2.5) Lemma. Let $X$ be a complete subvariety of $\widetilde{\mathcal{M}}_{g}$ of dimension $2 g-3$. Then $X$ contains points corresponding to chains of $g$ elliptic curves. In particular, $X$ intersects every component of the boundary $\widetilde{\mathcal{M}}_{g}-\mathcal{M}_{g}$.

Proof. Since $X$ is of maximal dimension, the inequalities in the proof of Lemma 2.4 are equalities. Some geometric consequences of this will be exploited here. We prove the statement by induction on $g$; the case $g=2$ is clear, so assume $g \geq 3$. We know that $X$ meets $\Delta_{i}$ for some $i$ with $0<i \leq g / 2$. The inverse image of $X \cap \Delta_{i}$ in $\widetilde{\mathcal{M}}_{i, 1} \times \widetilde{\mathcal{M}}_{g-i, 1}$ is pure of dimension $2 g-4$. Let $Z$ be a component. Let $A_{i, 1}$ be the projection on the first factor and let $B_{g-i, 1}$ be the projection on the second factor. Then $\operatorname{dim} A_{i, 1}=2 i-2$ and $\operatorname{dim} B_{g-i, 1}=2(g-i)-2$. It follows that $Z=A_{i, 1} \times B_{g-i, 1}$. When $i=1$, the factor $A_{1,1}$ corresponds to a single elliptic curve. The image $B_{g-1}$ in $\widetilde{\mathcal{M}}_{g-1}$ and, for $i \geq 2$, the images $A_{i}$ and $B_{g-i}$ in $\widetilde{\mathcal{M}}_{i}$ resp. $\widetilde{\mathcal{M}}_{g-i}$ contain points corresponding to smooth curves; this follows again from maximality. Hence $A_{i, 1}$ is the inverse image of $A_{i}$ for $i \geq 2$ and $B_{g-i, 1}$ is the inverse image of $B_{g-i}$ for all $i$. Now use induction: pick points in $A_{i}$ (for $i \geq 2$ ) and $B_{g-i}$ corresponding to chains of elliptic curves, and (for any $i$ ) pick points in $A_{i, 1}$ and $B_{g-i, 1}$ corresponding to marked points on extremal components of the chains. Gluing gives a chain of $g$ elliptic curves.

Remark. The proof above is very similar to the way Keel and Sadun deduce from [KS, Cor.1.2] that a complete $X$ of dimension $2 g-3$ in $\widetilde{\mathcal{M}}_{g}$ doesn't exist in characteristic 0 for $g \geq 3$.

Let $\mathcal{H}_{g}$ be the hyperelliptic locus in $\mathcal{M}_{g}$ and $\widetilde{\mathcal{H}}_{g}$ the hyperelliptic locus in $\widetilde{\mathcal{M}}_{g}$, that is, the closure of $\mathcal{H}_{g}$ in $\widetilde{\mathcal{M}}_{g}$. In what follows, we assume that the characteristic is different from 2. Then the theory of admissible covers [HM, §4] can be used to describe the stable hyperelliptic curves.

For $g \geq 1$, let $\left(D, b_{1}, \ldots, b_{2 g+2}\right)$ be a stable $(2 g+2)$-pointed curve of genus 0 . The dual graph of $D$ is a tree. Define a node of $D$ to be even (resp. odd) if the number of marked points on either side of the node is even (resp. odd). There exists a unique admissible cover $A$ of degree 2 of $D$. The inverse image of a component of $D$ is the unique double cover of that component that is ramified exactly over the marked points and the odd nodes on that component. (The double cover is disconnected exactly when the component contains no marked points and only even nodes.) The $2 g+2$ Weierstrass points on $A$ are by definition the inverse images of the $2 g+2$ marked points. The admissible cover is a nodal curve. It may contain smooth rational components that meet the rest of the curve in only 2 points. (Observe that these necessarily cover a component of $D$ with 2 marked points and 1 node.) A stable hyperelliptic curve of genus $g \geq 2$ is by definition the stable curve of genus $g$ obtained from the admissible cover by contracting those components. The Weierstrass points on a stable hyperelliptic curve are the images of the Weierstrass points on the admissible cover. Only the Weierstrass points on contracted components become singular points. When $g=1$, we remember the Weierstrass points and consider the admissible covers as stable 4-pointed curves of genus 1.

A stable hyperelliptic curve is of compact type if and only if all the nodes of the stable $(2 g+2)$-pointed curve $D$ of genus 0 are odd. No components are contracted and these curves may be identified with the admissible covers. Therefore $\widetilde{\mathcal{H}}_{g}$, considered as a coarse 
moduli space, may be identified for $g \geq 1$ with the quotient of an open set in $\overline{\mathcal{M}}_{0,2 g+2}$ (the complement of the 'even' boundary divisors) by the natural action of the symmetric group on $2 g+2$ letters.

(2.6) Lemma. (Char. $\neq 2$.) A complete subvariety of $\widetilde{\mathcal{H}}_{g}$ has dimension at most $g-1$. If $Z$ is a complete subvariety of dimension $g-1$ then $Z \cap \mathcal{H}_{g} \neq \emptyset$.

Proof. By induction on $g$, the case $g=1$ being trivial. Assume $g>1$ and let $Z$ be a positive-dimensional complete subvariety of $\widetilde{\mathcal{H}}_{g}$. Then $Z \cap \Delta_{i} \neq \emptyset$ for some $i$ with $0<i \leq g / 2$, since $\mathcal{H}_{g}$ is affine. Denote by $W \widetilde{\mathcal{H}}_{i} \subset \widetilde{\mathcal{H}}_{i, 1}$ the locus where the marked point is a Weierstrass point. There exists a finite and surjective map $W \widetilde{\mathcal{H}}_{i} \times W \widetilde{\mathcal{H}}_{g-i} \rightarrow \Delta_{i} \cap \widetilde{\mathcal{H}}_{g}$. Hence $\operatorname{dim} Z \leq 1+(i-1)+(g-i-1)=g-1$. If equality holds then $Z$ is not contained in the boundary.

In characteristic 0 a complete subvariety of $\widetilde{\mathcal{M}}_{g}$ of dimension $2 g-3$ doesn't exist for $g \geq 3$. In positive characteristic we have the following.

(2.7) Proposition. (Char. $>$ 2.) Let $X$ be a complete subvariety of $\widetilde{\mathcal{M}}_{g}$ of maximal dimension $2 g-3$, e.g., an irreducible component of $V_{0}\left(\overline{\mathcal{M}}_{g}\right)$.

(i) $X$ contains points corresponding to chains of $g$ elliptic curves such that on every nonextremal elliptic curve in the chain the two attachment points differ by a 2-torsion point.

(ii) $X$ contains points corresponding to smooth hyperelliptic curves.

Proof. The proof of the first statement is entirely analogous to the proof of Lemma 2.5; the only adaptation required is that one should choose points in $A_{i, 1}$ and $B_{g-i, 1}$ corresponding to marked points on extremal components of the chains that differ from the attachment point by a point of order 2 (such points exist). Fulton ([HM, p.88]) shows that the curves from (i) are stable hyperelliptic curves. Thus $X \cap \widetilde{\mathcal{H}}_{g}$ is complete of dimension at least $g-1$ and nonempty. We conclude by applying Lemma 2.6.

\section{$\S 3$. Relations between the tautological classes}

We work over an algebraically closed field of characteristic $\neq 2$. Let as before $R \mathcal{M}_{g}$ denote the moduli space of pairs $\left(C^{\prime}, C\right)$ with $C$ a smooth curve of genus $g$ and $C^{\prime}$ an étale double cover of $C$. We consider the Prym map

$$
P: R \mathcal{M}_{g} \longrightarrow \mathcal{A}_{g-1}, \quad\left(C^{\prime}, C\right) \mapsto P\left(C^{\prime}, C\right) .
$$

Here $P\left(C^{\prime}, C\right)$ is the Prym variety of $C^{\prime} \rightarrow C$, i.e., the identity component of the kernel of the norm map $\mathrm{Nm}: J\left(C^{\prime}\right) \rightarrow J(C)$. The Prym variety comes with a canonical principal polarization $\Xi$, see $[\mathrm{M} 1, \mathrm{p} .333]$.

The Prym map $P$ extends to a map

$$
P: R \widetilde{\mathcal{M}}_{g} \longrightarrow \mathcal{A}_{g-1}^{*}, \quad\left(C^{\prime}, C\right) \mapsto P\left(C^{\prime}, C\right)
$$

where $\mathcal{A}_{g-1}^{*}$ is the Satake compactification and $R \widetilde{\mathcal{M}}_{g}$ is the moduli space of pairs $\left(C^{\prime}, C\right)$ with $C$ a stable curve of genus $g$ of compact type and $C^{\prime}$ an étale double cover of $C$. Then 
$C^{\prime}$ is a stable curve of genus $2 g-1$, not necessarily of compact type. The Prym variety is then the identity component of the kernel of the norm map on the generalized Jacobians.

We establish a relation between the Chern classes of the Hodge bundle and the pullback of such classes under the Prym map. Consider the universal morphism of curves $q: C^{\prime} \rightarrow C$ over the moduli space $R \widetilde{\mathcal{M}}_{g}$. The universal curve $\pi: C=C_{g} \rightarrow R \widetilde{\mathcal{M}}_{g}$ carries a torsion line bundle $L$ defined by $q_{*}\left(O_{C^{\prime}}\right)=O_{C} \oplus L$ and $L^{\otimes 2} \simeq O_{C}$.

Let $\omega$ be the relative dualizing sheaf of $C_{g}$ over $R \widetilde{\mathcal{M}}_{g}$. We define the Prym-Hodge bundle $\mathbb{E}^{\prime}$ on $R \widetilde{\mathcal{M}}_{g}$ by

$$
\mathbb{E}^{\prime}:=\pi_{*}(\omega \otimes L)
$$

This is a vector bundle of rank $g-1$ on $R \widetilde{\mathcal{M}}_{g}$. We denote its Chern classes by $\lambda_{i}^{\prime}=c_{i}\left(\mathbb{E}^{\prime}\right)$, for $1 \leq i \leq g-1$.

(3.1) Theorem. Let $\phi: R \widetilde{\mathcal{M}}_{g} \rightarrow \widetilde{\mathcal{M}}_{g}$ be the map $\left(C^{\prime}, C\right) \mapsto C$. Then $\operatorname{ch}\left(\mathbb{E}^{\prime}\right)=$ $\operatorname{ch}\left(\phi^{*}(\mathbb{E})\right)-1$.

Proof. Applying the Grothendieck-Riemann-Roch theorem (GRR) to the line bundle $\omega \otimes L$ and the morphism $\pi$ gives

$$
\operatorname{ch}\left(\mathbb{E}^{\prime}\right)=\pi_{*}\left(\operatorname{ch}(\omega \otimes L) \cdot \operatorname{Td}^{\vee}(\omega)\right)
$$

Since we work in Chow groups tensored with $\mathbb{Q}$ we can disregard torsion classes and we may replace the term $\omega \otimes L$ on the right by $\omega$, so that

$$
\operatorname{ch}\left(\mathbb{E}^{\prime}\right)=\pi_{*}\left(\operatorname{ch}(\omega) \cdot \operatorname{Td}^{\vee}(\omega)\right)
$$

But applying GRR to $\omega$ and $\pi$ gives

$$
\operatorname{ch}(\pi ! \omega)=\operatorname{ch}\left(\phi^{*}(\mathbb{E})\right)-1=\pi_{*}\left(\operatorname{ch}(\omega) \cdot \operatorname{Td}^{\vee}(\omega)\right)
$$

so that by comparing (1) and (2) we find $\operatorname{ch}\left(\mathbb{E}^{\prime}\right)=\operatorname{ch}\left(\phi^{*}(\mathbb{E})\right)-1$ as required.

(3.2) Corollary. We have $\phi^{*}\left(\lambda_{i}\right)=\lambda_{i}^{\prime}$ for $i=1, \ldots, g-1$.

Note that it also follows that $\lambda_{g}$ vanishes in the Chow ring $A^{*}\left(\widetilde{\mathcal{M}}_{g}\right)$. This is compatible with the fact that it vanishes on $\mathcal{A}_{g}$.

Denote by $\psi: R \widetilde{\mathcal{M}}_{g} \rightarrow \overline{\mathcal{M}}_{2 g-1}$ the morphism $\left(C^{\prime}, C\right) \mapsto C^{\prime}$. Then $\psi^{*}\left(\mathbb{E}_{2 g-1}\right)=$ $\phi^{*}(\mathbb{E}) \oplus \mathbb{E}^{\prime}$, hence $\psi^{*}\left(\lambda_{1}\right)=2 \lambda_{1}^{\prime}$.

(3.3) Corollary. The Torelli maps $R \widetilde{\mathcal{M}}_{g} \rightarrow \mathcal{A}_{g}^{*}$ (resp. $\left.R \widetilde{\mathcal{M}}_{g} \rightarrow \mathcal{A}_{2 g-1}^{*}\right)$ sending $\left(C^{\prime}, C\right)$ to $J(C)$ (resp. $J\left(C^{\prime}\right)$ ) are constant on any complete connected algebraic subset of a fiber of the extended Prym map $P: R \widetilde{\mathcal{M}}_{g} \rightarrow \mathcal{A}_{g-1}^{*}$.

Proof. Clearly $P^{*}\left(\lambda_{1}\right)=\lambda_{1}^{\prime}$. This is zero on a fiber of $P$, hence the same holds for $\phi^{*}\left(\lambda_{1}\right)$ and $\psi^{*}\left(\lambda_{1}\right)$. These are pull-backs of ample classes on $\mathcal{A}_{g}^{*}$ resp. $\mathcal{A}_{2 g-1}^{*}$ via the Torelli maps. The result follows. 
(3.4) Corollary. The restriction of the Prym map to a complete subvariety $Y$ of $R \widetilde{\mathcal{M}}_{g}$ is quasi-finite on $Y \cap R \mathcal{M}_{g}$.

\section{$\S 4$. The locus $V_{0}\left(R \mathcal{M}_{g}\right)$}

We now consider in $R \mathcal{M}_{g}$ the locus $V_{0}\left(R \mathcal{M}_{g}\right)$ of étale double covers $\left(C^{\prime}, C\right)$ where the $p$-rank of $C^{\prime}$ is zero $(p>2)$. Here is our motivation for studying this locus. Consider $\mathcal{A}_{g}$ and a toroidal compactification $\overline{\mathcal{A}}_{g}$ of the type constructed in [FC, Ch.IV] (such that the Hodge bundle extends). The class $\lambda_{g}$ has zero intersection with all boundary classes (those from $\overline{\mathcal{A}}_{g}-\mathcal{A}_{g}$ ). If a positive multiple of $\lambda_{g}$ can be written as an effective sum of subvarieties not contained in the boundary, then all those subvarieties are complete subvarieties of $\mathcal{A}_{g}$ of codimension $g$. The locus $V_{0}$ is a (canonical) effective cycle of this type in positive characteristic [G, Thm.9.2], while in characteristic 0 such an effective cycle doesn't exist for $g \geq 3\left[\mathrm{KS}\right.$, Cor.1.2]. As we saw in $\S 2$, the situation for $\widetilde{\mathcal{M}}_{g}$ and its compactification $\overline{\mathcal{M}}_{g}$ is exactly analogous.

Now consider $\mathcal{M}_{g}$, with compactification $\overline{\mathcal{M}}_{g}$. The class $\lambda_{g} \lambda_{g-1}$ has zero intersection with all boundary classes [F, p.112] and appears to be the natural analogue of the class $\lambda_{g}$ for $\mathcal{A}_{g}$ and $\widetilde{\mathcal{M}}_{g}$ (cf. [FP, §0]). In the search for an effective representative of a multiple of $\lambda_{g} \lambda_{g-1}$, it seems natural to combine the $p$-rank zero requirement with a condition specific to curves. Certainly, the construction of the Prym variety $P\left(C^{\prime}, C\right)$ associated to an étale double cover $C^{\prime}$ of a curve $C$ plays an important role in the theory of curves and Jacobians. The Prym variety is a principally polarized abelian variety of dimension $g(C)-1$. The requirement that it have $p$-rank zero should then impose the desired $g-1$ additional conditions; or equivalently, one may combine the two requirements by asking that $C^{\prime}$ have $p$-rank zero. Note also that the class of $V_{0}\left(\overline{\mathcal{M}}_{2 g-1}\right)$ is a multiple of $\lambda_{2 g-1}$ and that $\psi^{*}\left(\lambda_{2 g-1}\right)=\phi^{*}\left(\lambda_{g} \lambda_{g-1}\right)$. This explains our interest in the locus $V_{0}\left(R \mathcal{M}_{g}\right)$.

(4.1) Proposition. Every irreducible component of $V_{0}\left(R \mathcal{M}_{g}\right)$ has dimension at least $g-2$. A complete irreducible component of $V_{0}\left(R \mathcal{M}_{g}\right)$ has dimension equal to $g-2$.

Proof. The first statement follows by applying Lemma 2.1 to the family of curves $C^{\prime}$ over $R \mathcal{M}_{g}$. The second statement follows then from Diaz-Looijenga.

Let as before $\psi: R \widetilde{\mathcal{M}}_{g} \rightarrow \overline{\mathcal{M}}_{2 g-1}$ be the morphism $\left(C^{\prime}, C\right) \mapsto C^{\prime}$. We define

$$
V_{0}\left(R \widetilde{\mathcal{M}}_{g}\right)=\psi^{-1}\left(V_{0}\left(\overline{\mathcal{M}}_{2 g-1}\right)\right) .
$$

Clearly, this contains $V_{0}\left(R \mathcal{M}_{g}\right)=\psi^{-1}\left(V_{0}\left(\mathcal{M}_{2 g-1}\right)\right)$, and every irreducible component of $V_{0}\left(R \widetilde{\mathcal{M}}_{g}\right)$ has dimension at least $g-2$, by Lemma 2.1 again. Observe that $V_{0}\left(R \widetilde{\mathcal{M}}_{g}\right)$ is complete: it is a closed sublocus of the complete locus $R V_{0}\left(\widetilde{\mathcal{M}}_{g}\right)$.

For $g=2$, all components of $V_{0}\left(R \widetilde{\mathcal{M}}_{g}\right)$ have the expected dimension $g-2=0$. In fact, from Corollary 3.3 and the identification $\widetilde{\mathcal{M}}_{2}=\mathcal{A}_{2}$, the Prym map $P$ is finite on $R V_{0}\left(\widetilde{\mathcal{M}}_{2}\right)$. In $\S 6$ we count the number of points of $V_{0}\left(R \mathcal{M}_{2}\right)$. It turns out that $V_{0}\left(R \mathcal{M}_{2}\right)$ is empty for $p=3$, but nonempty for $p \geq 5$.

For $g \geq 3$, the situation is more complicated. We first analyze the case $g=3$ in some detail. We have the following description of the components of $V_{0}\left(R \widetilde{\mathcal{M}}_{3}\right)$. 


\section{(4.2) Proposition.}

(i) All components of $V_{0}\left(R \mathcal{M}_{3}\right)$ have dimension equal to $g-2=1$.

(ii) For every pair $\left(D^{\prime}, D\right) \in V_{0}\left(R \mathcal{M}_{2}\right)$ and every supersingular elliptic curve $E$, there is a component of $V_{0}\left(R \widetilde{\mathcal{M}}_{3}\right)$ isomorphic to $D$. The curve $C_{p}$ corresponding to $p \in D$ is obtained by gluing $D$ and $E$ at $p$, while $C_{p}^{\prime}$ is obtained by gluing two copies of $E$ to $D^{\prime}$ at the two inverse images of $p$.

(iii) For every pair $\left(E^{\prime}, E\right) \in V_{0}\left(R \mathcal{M}_{1,1}\right)$ and every component $X$ of $V_{0}\left(\widetilde{\mathcal{M}}_{2}\right)$, there is a component of $V_{0}\left(R \widetilde{\mathcal{M}}_{3}\right)$ isomorphic to the universal curve over $X$ (considered as a subvariety of $\widetilde{\mathcal{M}}_{2,1}$ ). The curve $C$ corresponding to $D \in X$ and $p \in D$ consists of $E$ and $D$ glued at $p$, while $C^{\prime}$ is obtained by gluing two copies of $D$ to $E^{\prime}$ (the inverse images of $0 \in E$ are identified with the two points $p)$.

(iv) For every pair $\left(E^{\prime}, E\right) \in V_{0}\left(R \mathcal{M}_{1,1}\right)$ and every two supersingular elliptic curves $F$ and $G$, there is a component of $V_{0}\left(R \widetilde{\mathcal{M}}_{3}\right)$ isomorphic to $E$ (considered as a subvariety of $\left.\widetilde{\mathcal{M}}_{1,2}\right)$. The curve $C_{p}$ corresponding to $0 \neq p \in E$ consists of $E$ with $F$ and $G$ glued on at 0 and $p$, while $C_{p}^{\prime}$ consists of $E^{\prime}$ with two copies of both $F$ and $G$ glued on at the respective inverse images.

The components of type (ii)-(iv) are the components of $V_{0}\left(R \widetilde{\mathcal{M}}_{3}\right)$ not intersecting $R \mathcal{M}_{3}$.

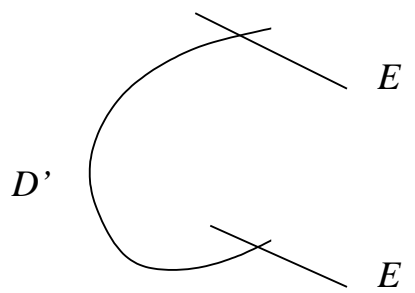

$D$

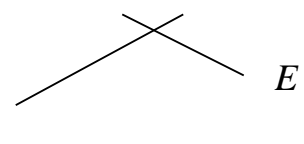

(ii)
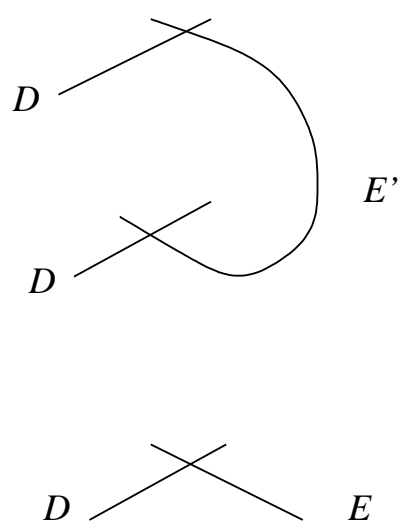

(iii)
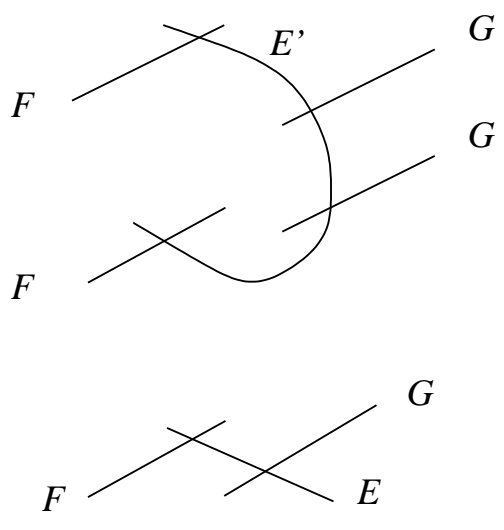

(iv)

(Figures (iii) and (iv) represent general points of the corresponding components.)

Proof. From Corollary 3.4, the restriction of the Prym map to $R V_{0}\left(\mathcal{M}_{g}\right)$ is quasi-finite. For $g=3$ this means that all components of $V_{0}\left(R \mathcal{M}_{3}\right)$ have dimension equal to $g-2=1$, the dimension of $V_{0}\left(\mathcal{A}_{2}\right)$. By taking the closure, we obtain the components of $V_{0}\left(R \widetilde{\mathcal{M}}_{3}\right)$ intersecting $R \mathcal{M}_{3}$. It is straightforward to check that (ii), (iii), and (iv) give a complete description of the $p$-rank zero étale double covers of reducible curves of genus 3 . Since these families are positive-dimensional, they yield the components of $V_{0}\left(R \mathcal{M}_{3}\right)$ not intersecting $R \mathcal{M}_{3}$.

The components of type (iii) have dimension 2 and provide the first examples of components of $V_{0}\left(R \widetilde{\mathcal{M}}_{g}\right)$ of dimension greater than $g-2$. Taking $X$ to be a component of $V_{0}\left(\widetilde{\mathcal{M}}_{g-1}\right)$, we find components of $V_{0}\left(R \widetilde{\mathcal{M}}_{g}\right)$ of dimension $2 g-4$.

Return to genus 3. From the description in Proposition 4.2, one might expect that all components of $V_{0}\left(R \mathcal{M}_{3}\right)$ are complete. However, this is not the case, as is shown by the 
following argument that we learned from Oort.

(4.3) Proposition. (Oort.) If $V_{0}\left(R \mathcal{M}_{g-1}\right)$ is nonempty, then it has a component of dimension $>g-3$ (necessarily noncomplete) or $V_{0}\left(R \mathcal{M}_{g}\right)$ has a noncomplete component.

Proof. Consider $\psi: R \widetilde{\mathcal{M}}_{g} \rightarrow \overline{\mathcal{M}}_{2 g-1}$ and the Torelli map $t: \overline{\mathcal{M}}_{2 g-1} \rightarrow \mathcal{A}_{2 g-1}^{*}$. The image $t \psi\left(R \widetilde{\mathcal{M}}_{g}\right)$ has dimension $3 g-3$; every component of the intersection with $V_{0}\left(\mathcal{A}_{2 g-1}\right)$ has dimension at least $g-2$. The intersection $V_{0}\left(\mathcal{A}_{2 g-1}\right) \cap t \psi\left(R \widetilde{\mathcal{M}}_{g}\right)$ equals $t \psi\left(V_{0}\left(R \widetilde{\mathcal{M}}_{g}\right)\right)$.

Let $Y$ be a component of $V_{0}\left(R \mathcal{M}_{g-1}\right)$. We may assume that $\operatorname{dim} Y=g-3$. Consider the following family in $V_{0}\left(R \widetilde{\mathcal{M}}_{g}\right)$ isomorphic to the universal curve over $\phi(Y)$ : for $\left(D^{\prime}, D\right) \in$ $Y$ and $p \in D$, construct $C$ by gluing a supersingular elliptic curve $E$ to $D$ at $p$ and $C^{\prime}$ by gluing two copies of $E$ to $D^{\prime}$ (just as in type (ii) above). Denote this family by $Z$. Then $t \psi(Z)$ has dimension $g-3$. Therefore it is strictly contained in a component of $t \psi\left(V_{0}\left(R \widetilde{\mathcal{M}}_{g}\right)\right)$. That component necessarily contains Jacobians of smooth curves $C^{\prime}$ and yields a noncomplete component of $V_{0}\left(R \mathcal{M}_{g}\right)$.

We may similarly consider a family of pairs $\left(C^{\prime}, C\right)$, where $C$ consists of two smooth components, $D$ of genus $i$ and $E$ of genus $j$, and $C^{\prime}$ consists of $D^{\prime}$ of genus $2 i-1$ and two copies of $E$. Let $\left(D^{\prime}, D\right)$ vary in a component $Y$ of $V_{0}\left(R \mathcal{M}_{i}\right)$ and $E$ in a component of $V_{0}\left(\mathcal{M}_{j}\right)$. Since the attachment points vary also, the dimension of this family equals $(\operatorname{dim} Y+1)+(2 j-2) \geq(i-1)+(2 j-2)=j+g-3$. (Mutatis mutandis, this includes the cases $i=1$ and $j=1$.) Denoting the family by $Z$, we have

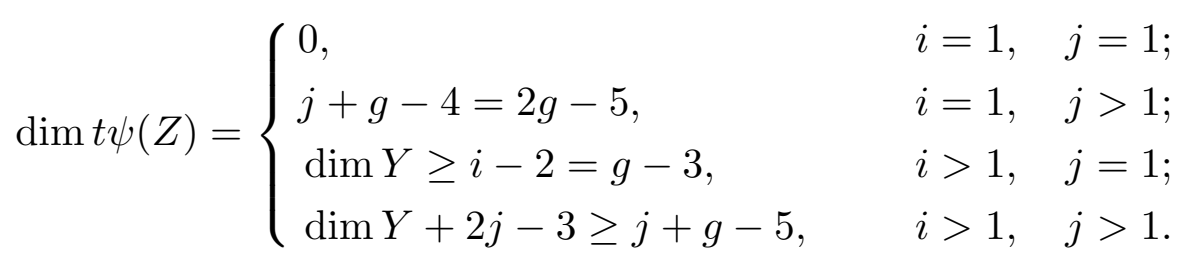

The proposition uses the case $j=1$ for $g \geq 3$. We see that for $g \geq 4$ one may also use the case $j=2$. This proves: if $V_{0}\left(R \mathcal{M}_{g-2}\right)$ is nonempty, then it has a component of excess dimension or $V_{0}\left(R \mathcal{M}_{g}\right)$ has a noncomplete component.

(4.4) Corollary. For $p \geq 5$, the locus $V_{0}\left(R \mathcal{M}_{g}\right)$ has a noncomplete component for $g=3$, 4 , and 5 .

Proof. Use that $V_{0}\left(R \mathcal{M}_{2}\right)$ is nonempty and of dimension 0 . Then $V_{0}\left(R \mathcal{M}_{3}\right)$ has a noncomplete component. It has dimension 1. Now use the proposition again for $g=4$ and the discussion above for $g=5$.

We study the case $p=3$ and $g=3$ in the next section.

\section{$\S 5$. The locus $V_{0}\left(R \mathcal{M}_{3}\right)$ for $p=3$}

We start by recalling some facts concerning hyperelliptic curves. Assume char $(k) \neq 2$. Every étale double cover $C^{\prime}$ of a hyperelliptic curve $C$ arises from splitting up the set $B$ of branch points of $C$ into two disjoint subsets of even cardinality:

$$
B=B_{1} \amalg B_{2} \text {. }
$$


Then $C^{\prime}$ is a Galois cover of $\mathbb{P}^{1}$ with Galois group $\mathbb{Z} / 2 \times \mathbb{Z} / 2$ and we have a diagram

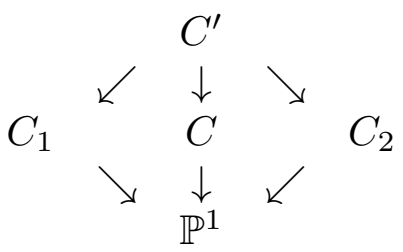

where $C_{1}$ (resp. $C_{2}$ ) is the hyperelliptic curve with branch points $B_{1}$ (resp. $B_{2}$ ). Moreover, the Prym variety of $C^{\prime}$ over $C$ is isomorphic to $J\left(C_{1}\right) \times J\left(C_{2}\right)$ (cf. [M1, p.346]). Note that $C^{\prime}$ is hyperelliptic if and only if $C_{1}$ or $C_{2}$ is a rational curve.

Next, assume that $k$ has characteristic $p>2$. The $p$-rank of a hyperelliptic curve $C$ can be computed easily. If $C$ is given by $y^{2}=f(x)$ with $f(x)$ a polynomial without multiple roots of degree $2 g+1$ or $2 g+2$, then the regular differentials $x^{i-1} d x / y$ with $1 \leq i \leq g$ form a basis. Write

$$
f(x)^{(p-1) / 2}=a(x)=\sum_{i=0}^{\infty} a_{i} x^{i}
$$

then the Hasse-Witt matrix with respect to this basis is

$$
H=\left(\begin{array}{cccc}
a_{p-1} & a_{2 p-1} & \ldots & a_{g p-1} \\
a_{p-2} & a_{2 p-2} & \ldots & a_{g p-2} \\
\vdots & \vdots & \ddots & \vdots \\
a_{p-g} & a_{2 p-g} & \ldots & a_{g p-g}
\end{array}\right)
$$

(Cf. [SV, p.54].) The $p$-rank of $C$ equals the semisimple rank of $H$. In particular, $C$ has $p$-rank 0 if and only if

$$
H \cdot H^{(p)} \cdot \ldots \cdot H^{\left(p^{g-1}\right)}=0,
$$

where $H^{\left(p^{i}\right)}$ is the matrix obtained from $H$ by raising every entry to the $\left(p^{i}\right)$ th power. This is independent of the chosen basis.

Let now $p=3$.

(5.1) Lemma. A hyperelliptic curve $C^{\prime}$ of genus 5 with a fixed-point-free involution cannot have 3-rank 0.

Proof. Let $C$ be the quotient curve of genus 3. Then $C$ is hyperelliptic and $C^{\prime}$ is an étale double cover of $C$. By the discussion above, the Prym variety of $\left(C^{\prime}, C\right)$ is the Jacobian of a curve $D$ of genus 2. Assume that $C^{\prime}$ has 3 -rank 0 . Then $D$ has 3 -rank 0 . Write $D$ as $y^{2}=\sum_{i=0}^{5} a_{i} x^{i}$. The Hasse-Witt matrix of $D$ equals

$$
H_{D}=\left(\begin{array}{ll}
a_{2} & a_{5} \\
a_{1} & a_{4}
\end{array}\right)
$$


We may assume $a_{5}=1$ and $a_{4}=0$. It follows then that $a_{1}=a_{2}=0$. We may assume $a_{0}=1$ and write $D$ as

$$
y^{2}=x^{5}+a x^{3}+1
$$

Then $C$ can be written as

$$
y^{2}=\left(x^{2}+b x+c\right)\left(x^{5}+a x^{3}+1\right)=x^{7}+b x^{6}+(a+c) x^{5}+a b x^{4}+a c x^{3}+x^{2}+b x+c
$$

and the Hasse-Witt matrix of $C$ equals

$$
H_{C}=\left(\begin{array}{ccc}
1 & a+c & 0 \\
b & a b & 1 \\
c & a c & b
\end{array}\right)
$$

with determinant $c^{2}-c b^{2}=c\left(c-b^{2}\right)$. The discriminant of $x^{2}+b x+c$ equals $b^{2}-c$, so it follows that $c=0$. Then

$$
H_{C}=\left(\begin{array}{ccc}
1 & a & 0 \\
b & a b & 1 \\
0 & 0 & b
\end{array}\right)
$$

and the $(3,3)$-entry of $H \cdot H^{(3)} \cdot H^{(9)}$ equals $b^{13}$. This forces $b=0$ and we obtain a contradiction.

(5.2) Proposition. For $p=3$, the locus $V_{0}\left(R \mathcal{M}_{3}\right)$ doesn't have a complete component.

Proof. Suppose instead that there is a complete one-dimensional family $X$ of smooth curves of genus 5, varying in moduli, such that each curve has 3-rank 0 and possesses a fixed-point-free involution. On $X$ the degree of $\lambda_{1}$ is positive. Hence the degree of the divisor $T$ in $\mathcal{M}_{5}$ of trigonal curves and their degenerations is positive, since $[T]=8 \lambda_{1}$ (cf. [HM, p.24]). It is easy to see that a trigonal curve of genus 5 cannot have a fixed-pointfree involution: the $g_{3}^{1}$ is unique, thus fixed by the involution; there are 2 fixed divisors; this forces fixed points on the curve. The intersection points of $X$ and $T$ come therefore from degenerate trigonal curves where the trigonal system decomposes as a $g_{2}^{1}$ plus a base point. These curves are hyperelliptic and Lemma 5.1 gives a contradiction.

(5.3) Example. For $p=5$, there do exist smooth hyperelliptic curves of genus 5 with 5-rank 0 and possessing a fixed-point-free involution. Consider $C$ of genus 3 given by

$$
y^{2}=\left(x^{2}-x+2\right)\left(x^{5}+x^{4}+2 x^{2}-2 x\right)=x^{7}+x^{5}-x^{4}+x^{3}+x^{2}+x .
$$

One easily checks that both $C$ and $D$, given by $y^{2}=f(x)=x^{5}+x^{4}+2 x^{2}-2 x$, have 5 -rank 0 . The étale double cover $C^{\prime}$ is given by

$y^{2}=f\left(\frac{x^{2}-2}{2 x-1}\right)(2 x-1)^{6}=2 x^{11}-2 x^{10}+x^{9}+x^{8}-x^{7}-2 x^{6}+2 x^{5}-x^{4}+x^{3}-2 x^{2}-2 x+2$,

with fixed-point-free involution $(x, y) \mapsto\left(\frac{x+1}{2 x-1},-y\right)$. 
(5.4) Example. For $p=3$, there do exist smooth hyperelliptic curves of genus 7 with 3-rank 0 and possessing a fixed-point-free involution. Consider $C$ of genus 4 given by

$$
y^{2}=x\left(x^{8}+x^{6}+x^{5}-x^{3}-x^{2}-1\right) .
$$

One easily checks that both $C$ and $D$, given by $y^{2}=f(x)=x^{8}+x^{6}+x^{5}-x^{3}-x^{2}-1$, have 3 -rank 0 . The étale double cover $C^{\prime}$ is given by $y^{2}=f\left(x^{2}\right)=x^{16}+x^{12}+x^{10}-x^{6}-x^{4}-1$, with fixed-point-free involution $(x, y) \mapsto(-x,-y)$.

(5.5) Example. For $p=3$, there do exist smooth hyperelliptic curves of genus 3 possessing an étale double cover with 3 -rank 0 . Consider $C$ of genus 3 given by

$$
y^{2}=\left(x^{3}+x+1\right)\left(x^{4}+x-1\right)=x^{7}+x^{5}-x^{4}-x^{3}+x^{2}-1 .
$$

One easily checks that $C$ and the elliptic curves $v^{2}=x^{3}+x+1$ and $w^{2}=x^{4}+x-1$ have 3 -rank 0 . The étale double cover $C^{\prime}$ is the normalization of

$$
v^{8}-w^{6}-v^{4} w^{2}-v^{6}-w^{4}-v^{4}+v^{2}-1=0,
$$

with fixed-point-free involution induced by $(v, w) \mapsto(-v,-w)$.

\section{$\S 6$. The number of $p$-rank zero étale double covers of genus 2 curves}

In this section we count the number of points of $V_{0}\left(R \mathcal{M}_{2}\right)$, that is, we count the number of pairs $\left(C^{\prime}, C\right)$ with $C$ a smooth curve of genus 2 and $C^{\prime}$ an étale double cover of $C$ with $p$-rank zero. Our method is to use intersection theory on stacks. In the terminology of Mumford [M2, p.293], we intersect the $Q$-classes of $R V_{0}\left(\widetilde{\mathcal{M}}_{2}\right)$ and $P^{-1} V_{0}\left(\mathcal{A}_{1}\right)$ inside $R \widetilde{\mathcal{M}}_{2}$. The intersection has dimension zero and the $Q$-class of every point is counted with the intersection multiplicity in the universal deformation space. This gives the number of points of $V_{0}\left(R \widetilde{\mathcal{M}}_{2}\right)$ and we need to subtract the contribution of the reducible curves to obtain the number of points of $V_{0}\left(R \mathcal{M}_{2}\right)$.

(6.1) Theorem. The weighted number of isomorphism classes of pairs $\left(C^{\prime}, C\right)$ with $C$ a smooth curve of genus 2 and $C^{\prime}$ an étale double cover of $C$ with p-rank zero is given by

$$
\sum_{\left(C^{\prime}, C\right)} \frac{m\left(C^{\prime}, C\right)}{\left|\operatorname{Aut}\left(C^{\prime}, C\right)\right|}=\frac{1}{384}(p-3)(p-1)^{2}(p+1),
$$

where $m\left(C^{\prime}, C\right) \in \mathbb{Z}_{\geq 1}$ is the intersection multiplicity in the universal deformation space and $\left|\operatorname{Aut}\left(C^{\prime}, C\right)\right|$ denotes the number of automorphisms of $C$ that fix the point of order 2 in $J(C)$ corresponding to $C^{\prime}$.

Proof. Since $V_{0}\left(\mathcal{A}_{1}\right)$ has class $(p-1) \lambda_{1}$, the class of $P^{-1} V_{0}\left(\mathcal{A}_{1}\right)$ equals

$$
(p-1) P^{*}\left(\lambda_{1}\right)=(p-1) \lambda_{1}^{\prime}=(p-1) \phi^{*} \lambda_{1},
$$


by Corollary 3.2. By [G, Thm.9.2], the class of $R V_{0}\left(\widetilde{\mathcal{M}}_{2}\right)$ equals $(p-1)\left(p^{2}-1\right) \phi^{*} \lambda_{2}$ (note that $\widetilde{\mathcal{M}}_{2}=\mathcal{A}_{2}$ as stacks). To evaluate the degree of the intersection, we apply $\phi_{*}$. We obtain

$$
\phi_{*}\left((p-1) \phi^{*} \lambda_{1} \cdot(p-1)\left(p^{2}-1\right) \phi^{*} \lambda_{2}\right)=15(p-1)^{2}\left(p^{2}-1\right) \lambda_{1} \lambda_{2}=\frac{1}{384}(p-1)^{2}\left(p^{2}-1\right),
$$

since $\lambda_{1} \lambda_{2}=\frac{1}{5760}$ on $\overline{\mathcal{M}}_{2}$. This is the weighted number of points of $V_{0}\left(R \widetilde{\mathcal{M}}_{2}\right)$.

The number of reducible curves in this intersection can be computed easily. We need an ordered pair $\left(E_{1}, E_{2}\right)$ of supersingular elliptic curves and a point of order 2 on $E_{1}$. We find

$$
3 \cdot \frac{p-1}{24} \cdot \frac{p-1}{24}=\frac{1}{192}(p-1)^{2} .
$$

In the next proposition we show that these pairs count with multiplicity $p+1$. Therefore the weighted number of points of $V_{0}\left(R \mathcal{M}_{2}\right)$ equals

$$
\begin{aligned}
\frac{1}{384}(p-1)^{2}\left(p^{2}-1\right)-\frac{1}{192}(p+1)(p-1)^{2} & =\frac{1}{384}(p-1)^{2}(p+1)((p-1)-2) \\
& =\frac{1}{384}(p-3)(p-1)^{2}(p+1),
\end{aligned}
$$

as required.

In $\S 7$ we give a number of examples illustrating this formula. Note that $V_{0}\left(R \mathcal{M}_{2}\right)$ is empty for $p=3$, but nonempty for $p \geq 5$.

(6.2) Proposition. The locus $R V_{0}\left(\widetilde{\mathcal{M}}_{2}\right)$ intersects $P^{-1} V_{0}\left(\mathcal{A}_{1}\right)$ with multiplicity $p+1$ at points corresponding to stable curves with two elliptic components.

Proof. For a smooth étale double cover $D^{\prime} \rightarrow D$ corresponding to a point $\eta$ of order 2 in $J(D)$ the codifferential of the Prym map $P: R \mathcal{M}_{g} \rightarrow \mathcal{A}_{g-1}$ is given by the multiplication map $\operatorname{Sym}^{2}\left(H^{0}\left(D, \omega_{D} \otimes \eta\right)\right) \rightarrow H^{0}\left(D, \omega_{D}^{2}\right)$. We extend this result to certain étale double covers of reducible curves of genus 2 in the following manner.

Let $C$ be a stable curve of genus 2 consisting of two elliptic components $E_{1}$ and $E_{2}$. We let $\operatorname{Def}(C)$ be the space of infinitesimal deformations of $C$. Since $\widetilde{\mathcal{M}}_{2}=\mathcal{A}_{2}$, it can be identified with the space of infinitesimal deformations of the Jacobian $E_{1} \times E_{2}$ of $C$ (as a principally polarized abelian surface). We thus get

$$
\operatorname{Def}(C) \cong \operatorname{Sym}^{2}\left(T_{E_{1}} \oplus T_{E_{2}}\right)=\operatorname{Sym}^{2}\left(T_{E_{1}}\right) \oplus\left(T_{E_{1}} \otimes T_{E_{2}}\right) \oplus \operatorname{Sym}^{2}\left(T_{E_{2}}\right),
$$

where $T_{E_{i}}$ denotes the tangent space to $E_{i}$ at the origin. Note that the tangent space and the cotangent space to $\mathcal{A}_{2}$ are identified via the principal polarization.

Consider now the étale double cover $C^{\prime}$ of $C$ defined by a point $\eta$ of order 2 of $E_{1}$. It consists of the étale double cover $E_{1}^{\prime}$ of $E_{1}$ defined by $\eta$ and two copies of $E_{2}$. Then

$$
H^{0}\left(C, \omega_{C} \otimes \eta\right)=H^{0}\left(E_{1}, \omega_{E_{1}} \otimes \eta\right) \oplus H^{0}\left(E_{2}, \omega_{E_{2}}\right)=H^{0}\left(E_{2}, \omega_{E_{2}}\right)=T_{E_{2}} .
$$

With these identifications, the codifferential of the Prym map becomes the inclusion

$$
\operatorname{Sym}^{2}\left(T_{E_{2}}\right) \rightarrow \operatorname{Sym}^{2}\left(T_{E_{1}}\right) \oplus\left(T_{E_{1}} \otimes T_{E_{2}}\right) \oplus \operatorname{Sym}^{2}\left(T_{E_{2}}\right)
$$


and the tangent space to the fiber of the Prym map is given by

$$
\operatorname{Sym}^{2}\left(T_{E_{1}}\right) \oplus\left(T_{E_{1}} \otimes T_{E_{2}}\right) .
$$

On the other hand, for a $C$ which is supersingular (i.e., both $E_{i}$ are supersingular) one can calculate the directions in the deformation space $\operatorname{Def}(C)$ along which supersingularity (or equivalently, the condition that the $p$-rank is 0 ) is preserved. According to a calculation by Oort [O2, p.258] we find the $p+1$ directions

$$
\left(\begin{array}{cc}
-\zeta^{p} & 1 \\
1 & \zeta
\end{array}\right) \in \operatorname{Sym}^{2}\left(T_{E_{1}} \oplus T_{E_{2}}\right)
$$

where $\zeta^{p+1}=-1$. Here the $i$ th coordinate corresponds to $T_{E_{i}}$. These tangent vectors are not contained in $\operatorname{Sym}^{2}\left(T_{E_{1}}\right) \oplus\left(T_{E_{1}} \otimes T_{E_{2}}\right)$.

\section{$\S 7$. Examples}

We now give a number of examples to illustrate the formula of the preceding section. In $\S 5$ we discussed how the $p$-rank of a hyperelliptic curve may be computed and how the étale double covers of hyperelliptic curves can be described. In the case where $g(C)=2$ we may assume that $C_{2}$ is a rational curve and that $C_{1}=E$ is an elliptic curve. The Prym variety of $\left(C^{\prime}, C\right)$ is isomorphic to $E$. Below, we will identify the étale double cover $C^{\prime}$ with a point $\eta$ of order 2 in $J(C)$. With $C$ defined by $y^{2}=f(x)$, we give $\eta$ by choosing 2 of the 6 branch points.

(7.1) $p=3$. Over $\overline{\mathbb{F}}_{3}$ the elliptic curve with $j=0=1728$ can be written $y^{2}=x^{3}-x$. Hence $C$ can be written $y^{2}=\left(x^{2}+a x+b\right)\left(x^{3}-x\right)$ with $\eta$ corresponding to $x^{2}+a x+b$. Then the Hasse-Witt matrix equals

$$
H=\left(\begin{array}{ll}
a_{2} & a_{5} \\
a_{1} & a_{4}
\end{array}\right)=\left(\begin{array}{ll}
-a & 1 \\
-b & a
\end{array}\right)
$$

and we have the condition $H \cdot H^{(p)}=0$. In particular, $0=\operatorname{det}(H)=b-a^{2}$; note that this condition already makes $C$ singular: $x^{2}+a x+a^{2}=(x-a)^{2}$. So, not only there isn't a smooth $C$ of genus 2 and 3-rank 0 with unramified double cover $C^{\prime}$ of 3-rank 0 (hence Prym of 3-rank 0), there isn't even a smooth $C$ of 3-rank $\leq 1$ with Prym of 3-rank 0.

(7.2) $p=5$. Over $\overline{\mathbb{F}}_{5}$ the elliptic curve with $p$-rank 0 has $j=0$ and can be written $y^{2}=x^{3}-1$. Hence $C$ can be written $y^{2}=\left(x^{2}+a x+b\right)\left(x^{3}-1\right)$ with $\eta$ corresponding to $x^{2}+a x+b$. Now $a(x)=f(x)^{2}$ and

$$
H=\left(\begin{array}{ll}
a_{4} & a_{9} \\
a_{3} & a_{8}
\end{array}\right) .
$$

One checks that $\operatorname{det}(H)=0$ gives $b=-a^{2}$ or $b=-2 / a$. Since $b=-a^{2}$ leads to a singular curve $\left(x^{2}+a x-a^{2}=(x-2 a)^{2}\right)$ one is left with $b=-2 / a$. Working out $H \cdot H^{(p)}=0$ leads to the necessary condition $a^{15}-a^{9}+1=0$; but $a^{3}+3=0$ is to be avoided, as it leads to 
a singular curve. The residual polynomial in $\mathbb{F}_{5}[a]$ of degree 12 has 3 irreducible factors of degree 4 . Now note that the automorphism group of the 4 -tuple $\left\{1, \zeta, \zeta^{2}, \infty\right\}$ (with $\zeta$ a primitive cube root of 1 ) is acting on the solution set, reflecting the choice of $x$-coordinate in writing the elliptic curve with $j=0$ as $y^{2}=x^{3}-1$. It is the group $A_{4}$, generated by the transformations $x \mapsto \zeta x$ and $x \mapsto(x+2) /(x-1)$. A calculation shows that the solution set is one $A_{4}$-orbit. So there is one isomorphism class of pairs $\left(C^{\prime}, C\right)$ with $C^{\prime}$ of 5 -rank 0 ; it is defined over $\mathbb{F}_{5}$ and has only the hyperelliptic automorphism $y \mapsto-y$ (this follows since the $A_{4}$-orbit has length 12 ). So the answer to the counting problem is

$$
\frac{1}{2}=\frac{2 \cdot 4 \cdot 4 \cdot 6}{384}
$$

as in the theorem; this proves that the multiplicity $m\left(C^{\prime}, C\right)=1$. Finally, one would of course like to have a model over $\mathbb{F}_{5}$. One can try to take $\eta=\{0, \infty\}$ although this is not guaranteed to work:

$$
y^{2}=x\left(x^{4}+b_{3} x^{3}+b_{2} x^{2}+b_{1} x+b_{0}\right)
$$

with the $b_{i} \in \mathbb{F}_{5}$. The Prym condition is just $b_{2}^{2}+2 b_{1} b_{3}+2 b_{0}=0$ wheras the condition for $C$ simplifies to $H^{2}=0$. A solution is (for instance):

$$
y^{2}=x\left(x^{4}+x^{3}+2 x+3\right) \quad \text { with } \quad H=\left(\begin{array}{ll}
4 & 2 \\
2 & 1
\end{array}\right)
$$

Remark. The locus $R V_{0}\left(\mathcal{M}_{2}\right)$ is nonsingular at a point $\left(C^{\prime}, C\right)$ with $a$-number $a(C)=1$, while it has multiplicity $p+1$ at a point with $a(C)=2$. If the intersection with $P^{-1} V_{0}\left(\mathcal{A}_{1}\right)$ is transversal, the multiplicity $m\left(C^{\prime}, C\right)$ equals 1 resp. $p+1$.

(7.3) $p=7$. Over $\overline{\mathbb{F}}_{7}$ we start with the form $y^{2}=\left(x^{2}+a x+b\right)\left(x^{3}-x\right)$. To find solutions one can proceed as follows. The 4 entries of $H \cdot H^{(p)}$ and $\operatorname{det}(H)$ are 5 polynomials in $a$ and $b$ that have to vanish simultaneously. Using Maple, one computes the resultants modulo 7 w.r.t. the variable $b$ (resp. $a$ ) of the determinant and each of the 4 entries without problem; taking the polynomial g.c.d. modulo 7 of the 4 resultants leads to a polynomial in $a$ (resp. $b$ ) of degree 65 in both cases. The multiple factors in the $a$-polynomial are $a^{19}(a+2)^{3}(a+5)^{3}$ reducing the number of possible $a$-values to 43 . The factors of the $b$-polynomial all have multiplicity at least 2 , with $(b+6)^{22}\left(b^{2}+3 b+1\right)^{4} b^{3}$ the factors with multiplicity $>2$, reducing the number of possible $b$-values to 20. Examining the singular solutions leads to the exclusion of $a= \pm 2$ and of $b=0$, which leaves us with 41 possible $a$-values and 19 possible $b$-values. Clearly, when $(a, b)$ is a solution, so is $(-a, b)$; more generally, the automorphism group of the 4-tuple $\{0,1,-1, \infty\}$, the group $D_{4}$, is acting on the solution set; the $x$-transformations $x \mapsto-x$ resp. $x \mapsto(x-1) /(x+1)$ have the effect $(a, b) \mapsto(-a, b)$ resp.

$$
(a, b) \mapsto\left(\frac{2 b-2}{b+1+a}, \frac{b+1-a}{b+1+a}\right)
$$

We examine the orbits of this action. It is clear that $(a, b)=(0,1)$ is the unique fixed point; it is in fact the unique solution with a non-trivial stabilizer. Here $C$ is the curve $y^{2}=x^{5}-x$ 
which has 48 automorphisms; $\eta=\{i,-i\}$; the pair $(C, \eta)$ has 16 automorphisms. Note that $a(C)=2$ (since $H=0)$; hence we expect to count $(C, \eta)$ with multiplicity $p+1=8$ for a total contribution of $8 / 16=1 / 2$. The pair $(C, \eta)$ is isomorphic to $(C,\{0, \infty\})$.

It is easy to check that the only solution with $a=0$ is $b=1$, and vice versa. For the solutions with $a$ nonzero, 40 possible $a$-values remain. These fall into 5 orbits. We find only 18 possible $b$-values since a 'ramification' occurs: to $b^{2}+3 b+1=0$ correspond 8 $a$-values (the roots of $\left.\left(a^{4}-a^{2}-1\right)\left(a^{4}-3 a^{2}-1\right)\right)$ instead of the expected 4 .

So we find 5 more solutions $(C, \eta)$, each having only the automorphisms $y \mapsto \pm y$; also, $a(C)=1$ for all five. Hence each contributes $1 / 2$, so that the answer to the counting problem is

$$
6 \cdot \frac{1}{2}=3=\frac{4 \cdot 6 \cdot 6 \cdot 8}{384} .
$$

Two of the $5 D_{4}$-orbits are given by a polynomial in $\mathbb{F}_{7}[a]$ of degree 16 (with 4 irreducible factors of degree 4$)$. Hence the 2 corresponding isomorphism classes $(C, \eta)$ are defined over $\mathbb{F}_{49}$ (a Galois orbit of length 2). With some work, one finds explicit representatives:

$$
\begin{cases}C: & y^{2}=x\left(x^{4}+x^{3}+(i+1) x^{2}+(3 i-2) x-i\right)=x\left(x^{2}+3 i-2\right)\left(x^{2}+x-2 i+3\right) \\ \eta: & x^{2}+x-2 i+3 \\ E: & y^{2}=x\left(x^{2}+3 i-2\right) \quad(j=1728=-1)\end{cases}
$$

with $i=\sqrt{-1} \in \mathbb{F}_{49}$.

The other three $D_{4}$-orbits are given by a polynomial in $\mathbb{F}_{7}[a]$ of degree 24 (with 4 irreducible factors of degree 6$)$. The 3 corresponding isomorphism classes $(C, \eta)$ are defined over $\mathbb{F}_{343}$ (a Galois orbit of length 3 ). One eventually finds explicit representatives:

$$
\begin{cases}C: & y^{2}=\left(x^{2}+1\right)\left(x^{4}+x^{3}+\left(\rho^{2}+\rho+2\right) x^{2}+\left(3 \rho^{2}-3 \rho+1\right) x+\left(5 \rho^{2}-3 \rho+3\right)\right) \\ \eta: & x^{2}+1 \\ E: & y^{2}=x^{4}+x^{3}+\left(\rho^{2}+\rho+2\right) x^{2}+\left(3 \rho^{2}-3 \rho+1\right) x+\left(5 \rho^{2}-3 \rho+3\right) \quad(j=1728)\end{cases}
$$

with $\rho=\sqrt[3]{2} \in \mathbb{F}_{343}$.

(7.4) $p=11$. There are two cases: (A) the Prym variety is the elliptic curve with $j=1728$; we take $y^{2}=\left(x^{3}-x\right)\left(x^{2}+a x+b\right)$ as $(C, \eta)$; (B) the Prym is the elliptic curve with $j=0$; we work with $y^{2}=\left(x^{3}-1\right)\left(x^{2}+a x+b\right)$.

In case (A) one initially finds a polynomial in $a$ of degree 340; only its 5 linear factors come with (varying) multiplicities; the reduced polynomial has degree 197. The initial polynomial in $b$ also has degree 340; every factor has at least multiplicity 2 , and only its 4 linear factors have higher multiplicities; the reduced polynomial has degree 100. As it turns out, the linear factors lead either to singular curves or to the 4 solutions $(a, b)=(0,2),(0,6)$, $(3,1),(8,1)$. These 4 solutions form a single $D_{4}$-orbit; hence the corresponding pair $(C, \eta)$ has 4 automorphisms. One easily checks that $a(C)=2$ so that the expected contribution is $(p+1) / 4=12 / 4=3$. The remaining 192 solutions of the reduced polynomial in $a$ form 24 orbits; their expected contribution is $24 / 2=12$, making the total expected contribution in case (A) equal to 15 . The Galois orbits have lengths $1,4,4,4,6$, and 6 . 
In case (B) one initially finds a polynomial in a of degree 270; only a linear and a quadratic factor come with a multiplicity; the reduced polynomial has degree 243. Exactly the same happens for the polynomial in $b$. As it turns out, the linear and the quadratic factor lead to singular curves. The remaining 240 solutions of the reduced polynomial in $a$ form $20 A_{4}$-orbits; the expected contribution in case (B) is $20 / 2=10$. The Galois orbits have lengths $3,3,4$, and 10 .

Hence the total expected contribution equals 25, as in Theorem 6.1. So all intersections are transversal.

(7.5) $p=13$. Here we find the first example of a non-transversal intersection. We work with $y^{2}=\left(x^{3}+x+4\right)\left(x^{2}+a x+b\right)$ with Prym variety the supersingular elliptic curve $y^{2}=x^{3}+x+4$. One initially finds a polynomial in $a$ of degree 466; a linear and a quadratic factor come with multiplicity 15 and yield singular curves; the factor $a^{4}+8 a^{3}+7 a^{2}+8 a+9$ comes with multiplicity 2 ; one linear factor doesn't give a solution; the remaining reduced polynomial has degree 416 . The remaining polynomial in $b$ has degree 416 as well. The group $D_{2}$ acts; if all intersections were transversal and all points of intersection had $a$-number 1 , then the total contribution would be $104 / 2=52$. However, the answer is 105/2 according to Theorem 6.1. The only possible explanation is that exactly one of the intersections is non-transversal and has multiplicity 2. Indeed, the factor $a^{4}+8 a^{3}+7 a^{2}+8 a+9$ yields that intersection point. The pair $(C, \eta)$ is defined over $\mathbb{F}_{13}$; an equation is

$$
y^{2}=x^{5}+x^{4}+6 x^{3}-2 x^{2}+2 x
$$

with $\eta=\{0, \infty\}$. This is a nonsingular point of $R V_{0}\left(\mathcal{M}_{2}\right)$ and the tangent line may be given as

$$
y^{2}=x^{5}+x^{4}+(t+6) x^{3}+(2 t-2) x^{2}+(2-t) x
$$

with the same $\eta$. The moving elliptic curve is

$$
y^{2}=x^{4}+x^{3}+(t+6) x^{2}+(2 t-2) x+2-t
$$

and the coefficient of $x^{12}$ in $\left(x^{4}+x^{3}+(t+6) x^{2}+(2 t-2) x+2-t\right)^{6}$ is $t^{2}$ times a unit, which proves the multiplicity statement.

(7.6) $p=17$. There are two supersingular $j$-invariants. For the elliptic curve $y^{2}=x^{3}+x+1$ we find a Galois orbit of length 2 with $a$-number 2 and 4 automorphisms, contributing $2(p+1) / 4=9$, and a pair $(C, \eta)$ defined over $\mathbb{F}_{17}$ giving a simple tangency and contributing 1. The remaining solutions contribute $928 / 8=116$ for a total contribution of 126 .

For the elliptic curve $y^{2}=x^{3}-1$ we find the first example of a non-transversal intersection with $a$-number 2 . This proves that the intersection multiplicity cannot be read off from the Hasse-Witt matrix. Let the pair $(C, \eta)$ be given by

$$
y^{2}=\left(x^{3}-1\right)\left(x^{2}-2 x-2\right) \text {. }
$$

The 18 tangent directions to $R V_{0}\left(\mathcal{M}_{2}\right)$ can be written down explicitly. Two of them lie in the tangent plane to the Prym fiber and it turns out that they yield simple tangencies. The intersection multiplicity equals 20 . The $A_{4}$-orbit of this pair has length 3 and it 
contributes $3 \cdot 20 / 24=5 / 2$. There is also an $A_{4}$-orbit of length 12 with $a$-number 2 , contributing $12 \cdot 18 / 24=9$. The remaining solutions contribute $732 / 24=61 / 2$. The total contribution for $y^{2}=x^{3}-1$ equals 42 .

Adding up the contributions for the two elliptic curves, we obtain 168, as in Theorem 6.1 .

Acknowledgements. We thank Frans Oort for several useful discussions; Proposition 4.3 is due to him. We thank the Göran Gustafsson Stiftelse for support of the second author's visit to KTH in March 2001. The first author thanks Princeton University and the Swedish Research Council for support. Finally, we thank the referee for several useful comments.

\section{References}

[D1] S. Diaz: A bound on the dimensions of complete subvarieties of $\mathcal{M}_{g}$. Duke Math. J. 51 (1984), 405-408.

[D2] S. Diaz: Complete subvarieties of the moduli space of smooth curves. In Algebraic geometry, Bowdoin 1985 (S. Bloch, ed.), 77-81, Proc. Sympos. Pure Math., 46, Part 1, Amer. Math. Soc., Providence, RI, 1987.

[F] C. Faber: A conjectural description of the tautological ring of the moduli space of curves. In Moduli of curves and abelian varieties (C. Faber and E. Looijenga, eds.), 109129, Aspects Math., E33, Vieweg, Braunschweig, 1999.

[FL] C. Faber and E. Looijenga: Remarks on moduli of curves. In Moduli of curves and abelian varieties (C. Faber and E. Looijenga, eds.), 23-45, Aspects Math., E33, Vieweg, Braunschweig, 1999.

[FP] C. Faber and R. Pandharipande: Logarithmic series and Hodge integrals in the tautological ring. With an appendix by Don Zagier. Michigan Math. J. 48 (2000), 215-252.

[FC] G. Faltings and C.-L. Chai: Degeneration of abelian varieties. Ergebnisse der Mathematik und ihrer Grenzgebiete 22. Springer-Verlag, Berlin, 1990. xii+316 pp.

[G] G. van der Geer: Cycles on the moduli space of abelian varieties. In Moduli of curves and abelian varieties (C. Faber and E. Looijenga, eds.), 65-89, Aspects Math., E33, Vieweg, Braunschweig, 1999.

[GO] G. van der Geer and F. Oort: Moduli of abelian varieties: a short introduction and survey. In Moduli of curves and abelian varieties (C. Faber and E. Looijenga, eds.), 1-21, Aspects Math., E33, Vieweg, Braunschweig, 1999.

[HM] J. Harris and D. Mumford: On the Kodaira dimension of the moduli space of curves. With an appendix by William Fulton. Invent. Math. 67 (1982), 23-88.

[Ka] N. Katz: Slope filtration of F-crystals. Journées de Géométrie Algébrique de Rennes (Rennes, 1978), Vol. I, 113-163, Astérisque No. 63 (1979).

[KS] S. Keel and L. Sadun: Oort's conjecture for $\mathcal{A}_{g}$. Preprint 2002, math.AG/0204229, to appear in J. Amer. Math. Soc.

[Ko] N. Koblitz: $p$-adic variation of the zeta-function over families of varieties defined over finite fields. Compositio Math. 31 (1975), 119-218.

[L] E. Looijenga: On the tautological ring of $\mathcal{M}_{g}$. Invent. Math. 121 (1995), 411-419.

[M-B] L. Moret-Bailly: Pinceaux de variétés abéliennes. Astérisque No. 129 (1985). 
[M1] D. Mumford: Prym varieties I. In Contributions to analysis (a collection of papers dedicated to Lipman Bers), 325-350. Academic Press, New York, 1974.

[M2] D. Mumford: Towards an enumerative geometry of the moduli space of curves. In Arithmetic and geometry (M. Artin and J. Tate, eds.), Vol. II, 271-328, Progr. Math., 36, Birkhäuser, Boston, 1983.

[NO] P. Norman and F. Oort: Moduli of abelian varieties. Ann. of Math. 112 (1980), 413-439.

[O1] F. Oort: Subvarieties of moduli spaces. Invent. Math. 24 (1974), 95-119.

[O2] F. Oort: Hyperelliptic supersingular curves. In Arithmetic algebraic geometry (Texel, 1989) (G. van der Geer, F. Oort, J. Steenbrink, eds.), 247-284, Progr. Math., 89, Birkhäuser, Boston, 1991.

[O3] F. Oort: Complete subvarieties of moduli spaces. In Abelian varieties (Egloffstein, 1993) (W. Barth, K. Hulek, H. Lange, eds.), 225-235, de Gruyter, Berlin, 1995.

[SV] K.-O. Stöhr and J.F. Voloch: A formula for the Cartier operator on plane algebraic curves. J. Reine Angew. Math. 377 (1987), 49-64.

Institutionen för Matematik

Kungliga Tekniska Högskolan

S-100 44 Stockholm, Sweden

faber@math.kth.se

Korteweg-de Vries Instituut

Universiteit van Amsterdam

Plantage Muidergracht 24

NL-1018 TV Amsterdam, The Netherlands

geer@science.uva.nl 